\title{
New Squaring and Square-rooting Circuits Using Cdba
}

\author{
J. K. Pathak ${ }^{1}$, A. K. Singh ${ }^{2}$, Raj Senani ${ }^{3, *}$ \\ ${ }^{1}$ Department of Electronics and Communication Engineering, Echelon Institute of Technology, Faridabad, India \\ ${ }^{2}$ Department of Electronics and Communication Engineering, Faculty of Engineering and Technology, Sharda University, Ghaziabad, India \\ ${ }^{3}$ Division of Electronics and Communication Engineering, Netaji Subhas Institute of Technology, Sector 3, Dwarka, New Delhi, India \\ *Corresponding author: senani@ieee.org
}

Received September 19, 2014; Revised December 08, 2014; Accepted December 18, 2014

\begin{abstract}
One new squaring and two new square-rooting circuits based on CDBAs have been introduced. The new squarer circuit consists of a CDBA, two NMOS transistors and one resistor. On the other hand, the first squarerooting circuit consists of two CDBAs, two NMOS transistors while the second square- rooting circuit consists of only a single CDBA, two NMOS transistors and two PMOS transistors. The proposed circuits exhibit wide input voltage range, very small error and offer low output impedance to facilitate easy cascading without requiring additional buffers. PSPICE simulation results are included which confirm the practical workability of the new circuits.
\end{abstract}

Keywords: squarer, square-rooter, current differencing buffered amplifier

Cite This Article: J. K. Pathak, A. K. Singh, and Raj Senani, "New Squaring and Square-rooting Circuits Using Cdba." American Journal of Electrical and Electronic Engineering, vol. 2, no. 6 (2014): 175-179. doi: 10.12691/ajeee-2-6-4.

\section{Introduction}

Squaring and square-rooting circuits are widely used for continuous-time signal processing in measurement and instrumentation systems [1]. In order to maintain compatibility with existing voltage processing circuits as well as taking advantages [2] of the current-mode circuits, the new active element called Current differencing buffered amplifier (CDBA) [3] has received significant attention in literature since it can operate in both currentmode as well as voltage- mode. In earlier literature, the CDBA has been shown to offer a lot of flexibility in linear analog circuit design $[3,4,5,6]$. However, the use of CDBA in realizing non-linear functions has rather been limited $[7,8,9]$.

The purpose of this paper is to present new squaring and square-rooting circuits based on CDBAs. The proportionality constant of the new circuits can be controlled by the value of an external resistor. The effectiveness of the proposed circuits is verified through SPICE simulations.

\section{The Current-differencing Buffered Amplifier (CDBA)}

A practical CDBA can be described by the following relationships that take into account the various nonidealities:

$$
V_{p}=V_{n}=0, I_{Z}=\beta_{p} I_{p}-\beta_{n} I_{n}, V_{W}=\alpha V_{Z}
$$

where $\beta_{\mathrm{p}}=1-\varepsilon_{\mathrm{p}}$ and $\varepsilon_{\mathrm{p}}\left(1 \varepsilon_{\mathrm{p}} \mathrm{p}<<1\right)$ is the current tracking error from p-terminal to z-terminal, $\beta_{\mathrm{n}}=1-\varepsilon_{\mathrm{n}}$ and $\varepsilon_{\mathrm{n}}\left(1 \varepsilon_{\mathrm{n}} \mathrm{l}<<1\right)$ is the current tracking error from n-terminal to z-terminal, and $\alpha=1-\varepsilon_{\mathrm{v}}$ and $\varepsilon_{\mathrm{v}}\left(1 \varepsilon_{\mathrm{v}} \mathrm{l}<<1\right)$ is the voltage-tracking error from z-terminal to w-terminal of the CDBA. The circuit symbol of the CDBA is shown in Figure 1.

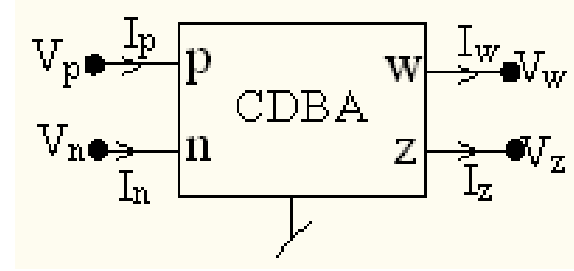

Figure 1. Symbolic notation of the CDBA

\section{Squarer Circuit}

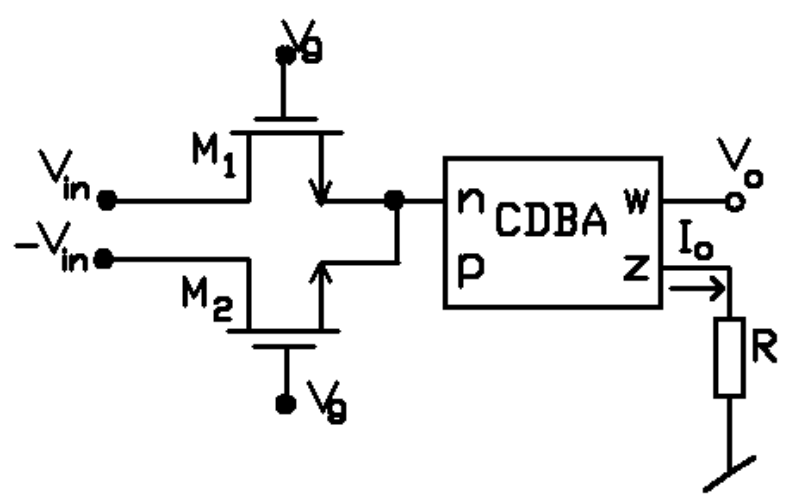

Figure 2. The proposed squarer circuit

Figure 2 shows the proposed squarer circuit. Assuming matched MOS transistors operating in triode region, a straight forward analysis of the circuit gives 


$$
V_{o}=\frac{\alpha \beta_{n} K W R V_{i n}^{2}}{L}=\frac{V_{i n}^{2}}{V_{r e f}}
$$

where $\mathrm{V}_{\text {ref }}$ is the proportionality constant, $(W / L)$ is the aspect ratio of MOS transistors, $K=\mu_{n} C_{o X}$ is the process trans-conductance parameter of the MOS transistor. The proportionality constant $\mathrm{V}_{\text {ref }}$ can be controlled by resistor $R$.

The output current $I_{o}$ is given by

$$
I_{o}=\frac{V_{o}}{R}=\frac{\beta_{n} K W V_{i n}^{2}}{L}=d V_{i n}^{2}
$$

where $d$ is the proportionality constant. Thus, the circuit can work in trans-admittance mode also.

\section{Square-rooting Circuits}

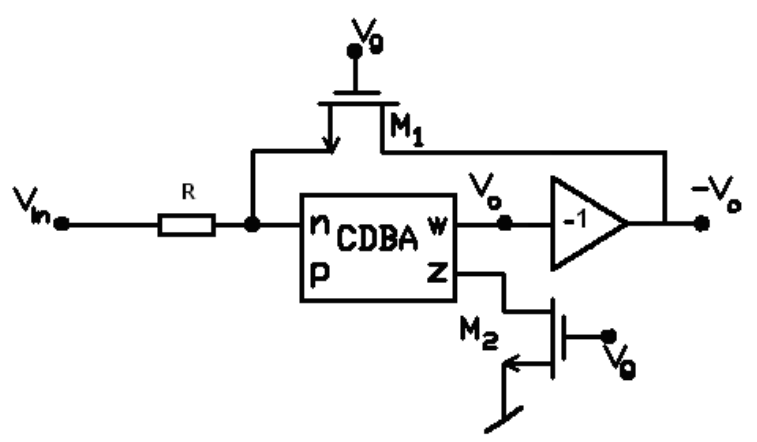

Figure 3. The first proposed square-rooting circuit

Figure 3 shows the first proposed square-rooting circuit. Assuming MOS transistors $\mathrm{M}_{1}$ and $\mathrm{M}_{2}$ to be matched and operating in triode region, the output voltage is found to be

$$
V_{o}=\sqrt{\frac{2 L_{1} V_{i n}}{K W_{1} R \beta_{n}\left(1+\beta_{n} \alpha^{2}\right)}}=b \sqrt{V_{\text {in }}} \text { for } V_{i n}>0
$$

where $\boldsymbol{b}$ is the proportionality constant, $(W / L)$ is the aspect ratio of MOS transistors, $K=\mu_{n} C_{o x}$ is the process transconductance of the MOS transistor and the proportionality constant $\boldsymbol{b}$ can be controlled by the resistor $R$. It is interesting to point out that the same circuit can work for the negative value of $V_{\text {in }}$ if we connect $R$ to p-terminal instead of n-terminal of the CDBA.

In the derivation of equation (4), to enable the cancellation of the non-linear terms, $L_{1}=L_{2}$ and $W_{2}=\alpha^{2} \beta_{n}{ }^{2} W_{1}$ have been taken where $\left(W_{1} / L_{1}\right)$ and $\left(W_{2} / L_{2}\right)$ are the aspect ratios of the MOS transistors $M_{1}$ and $M_{2}$ respectively.

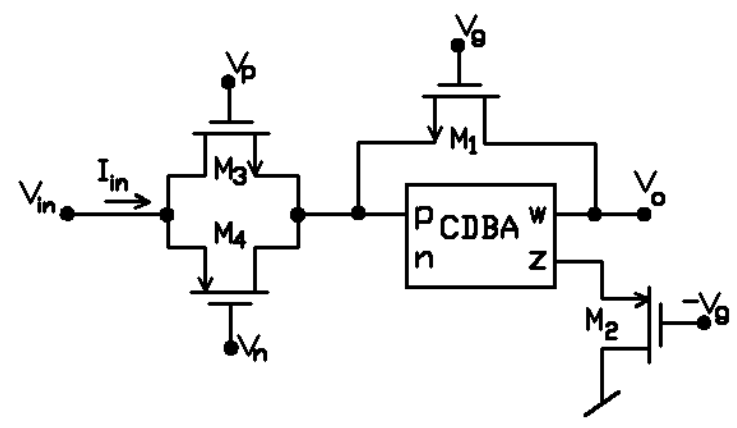

Figure 4, The second proposed square rooting circuit
Figure 4 shows the second proposed square-rooting in which the MOS transistors $\mathrm{M}_{1}$ and $\mathrm{M}_{2}$ are operating in triode region and are used to obtain the basic squarerooting function. The MOS transistors $\mathrm{M}_{3}$ and $\mathrm{M}_{4}$ are also operating in the triode region and are used to realize floating voltage controlled resistor [10]. After taking the model parameters of the CMOS process from [11], $V_{g}=2.63 \mathrm{~V}, L_{1}=L_{2}, W_{1}=0.2455 W_{2}$, a routine analysis of the proposed circuit then gives the following expression for its output voltage

$$
V_{o}=\sqrt{\frac{2 \alpha^{2} \beta_{p} L_{1} V_{i n}}{R_{e q}\left(K_{p} W_{2}+K_{n} W_{1} \beta_{p} \alpha^{2}\right)}}=e \sqrt{V_{i n}} \text { for } V_{i n}>0 \text { (5) }
$$

where $\boldsymbol{e}$ is the proportionality constant, $\left(W_{1} / L_{1}\right)$ and $\left(W_{2} / L_{2}\right)$ are the aspect ratios of MOS transistors $\mathrm{M}_{1}$ and $\mathbf{M}_{2}$ respectively, $K_{n}=\mu_{n} C_{o x}$ and $K_{p}=\mu_{p} C_{o x}$ being the process transconductances of the NMOS and PMOS transistor respectively.

Here, the proportionality constant $\boldsymbol{e}$ can be controlled by the resistor $R_{e q}$ which is given by

$$
R_{e q}=\frac{V_{\text {in }}}{I_{\text {in }}}=\frac{L_{3}}{K W_{3}\left(V_{n}-V_{p}-V_{t n}+V_{t p}\right)}
$$

where $\left(W_{3} / L_{3}\right)$ is the aspect ratio of the MOS transistor $M_{3}$, $V_{n}$ and $V_{p}$ are the gate voltages of $\mathrm{M}_{3}$ and $\mathrm{M}_{4}$ respectively, $V_{t n}$ and $V_{t p}$ are the threshold voltage of $\mathrm{M}_{3}$ and $\mathrm{M}_{4}$ respectively. To cancel non-linear terms, $W_{4}$ is taken equal to $\mu_{n} W_{3} / \mu_{p}$.

The same circuit can work for the negative value of $V_{\text {in }}$ if we connect the CMOS transistors constituting $R_{e q}$ to the n-terminal of the CDBA instead of the p-terminal.

\section{SPICE Simulation Results}

To verify the theoretical results, the squarer circuit has been simulated in SPICE using the CDBA from [11] operating at $\pm 5 \mathrm{~V}$ DC supply with $0.35 \mu \mathrm{m}$ CMOS process parameters. The value of $\mathrm{R}$ is made equivalent to $\frac{L}{10 \alpha \beta_{n} K W}$ so that proportionality constant becomes equal to 10 .

Using SPICE, the values of $\alpha, \beta_{p}$ and $\beta_{n}$ of the CDBA were found to be 1.0000998, 1.002501 and 1.002501 respectively. With $\mathrm{L}_{1}=\mathrm{L}_{2}=28 \mu \mathrm{m}, \mathrm{W}_{1}=\mathrm{W}_{2}=35 \mu \mathrm{m}$, the value of $R$, to make proportionality constant equal to 10 , has been adjusted to $990 \Omega$. Figure 5 shows the DC transfer characteristics of the proposed squarer circuit. Figure 6 shows the transient response of the circuit to a sinusoidal input of $4 \mathrm{~V}_{\mathrm{pp}}$, having zero average value and frequency of $1 \mathrm{kHz}$.

The square rooting circuit 1 of Figure 3 has been simulated in SPICE using the CDBA [11] operating at \pm 5 Volts DC supply with $0.35 \mu \mathrm{m}$ CMOS process parameters. In the simulations the value of $R$ is made equivalent to $\frac{2 L_{1}}{W_{1} \beta_{n} K\left(1+\beta_{n} \alpha^{2}\right)}$ so that the proportionality constant $\boldsymbol{b}$ becomes equal to 1 . To realize unity gain inverting amplifier, a CDBA-based inverter has been used. By 
selecting $L_{1}=L_{2}=21 \mu \mathrm{m}, W_{1}=21 \mu \mathrm{m}$ then $W_{2}=\alpha^{2} \beta_{n}{ }^{2} W_{1}$ comes out to be approximately equal to $21 \mu \mathrm{m}$. To get unity proportionality constant $\boldsymbol{b}$, the value of $\mathrm{R}$ was adjusted to $12 \mathrm{k} \Omega$. Figure 7 shows the DC transfer characteristics of the proposed square-rooting circuit. Figure 8 shows the transient response of the circuit to a sinusoidal input of $1 \mathrm{~V}_{\mathrm{pp}}$, average of 0 Volts and frequency of $1 \mathrm{kHz}$.

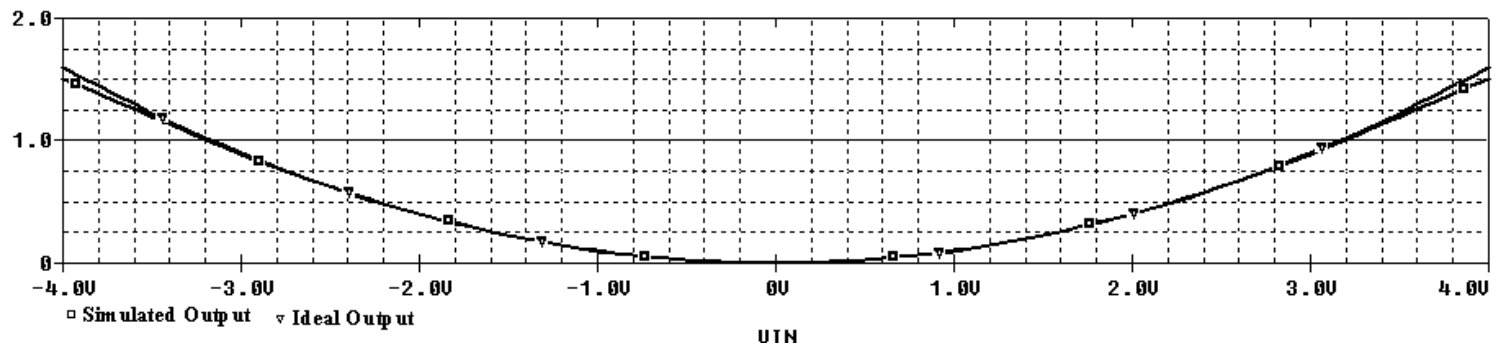

Figure 5. DC transfer characteristics of the proposed Squarer Circuit for $\mathrm{R}=990 \Omega$

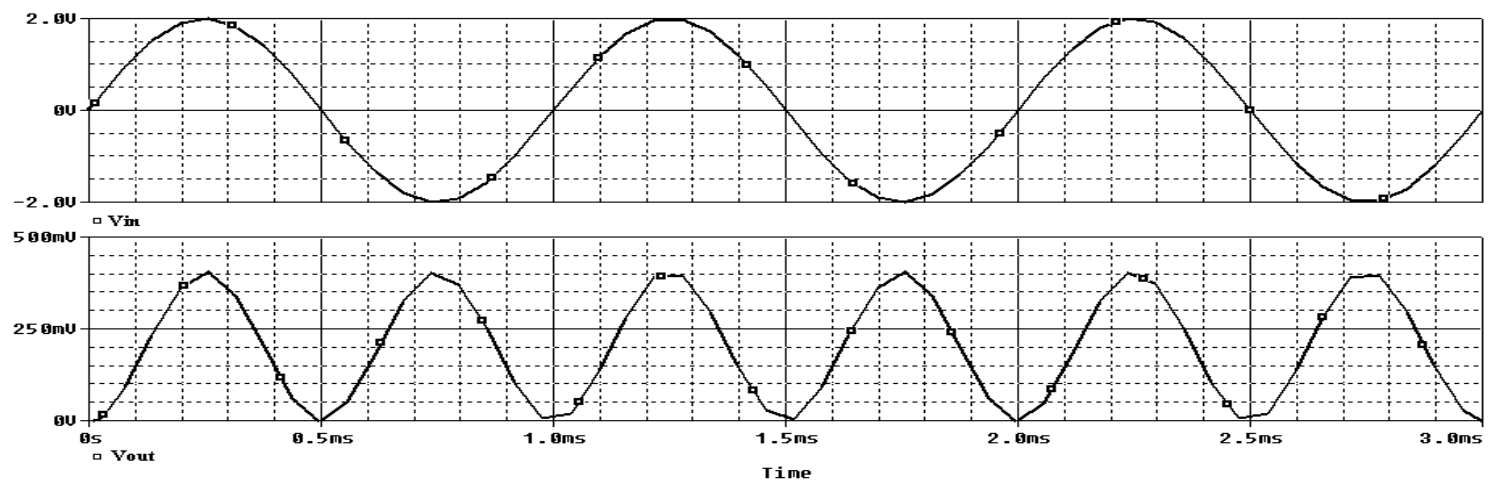

Figure 6. Output voltage waveform obtained from the proposed Squarer circuit for $1 \mathrm{KHz}$ Sinusoidal input

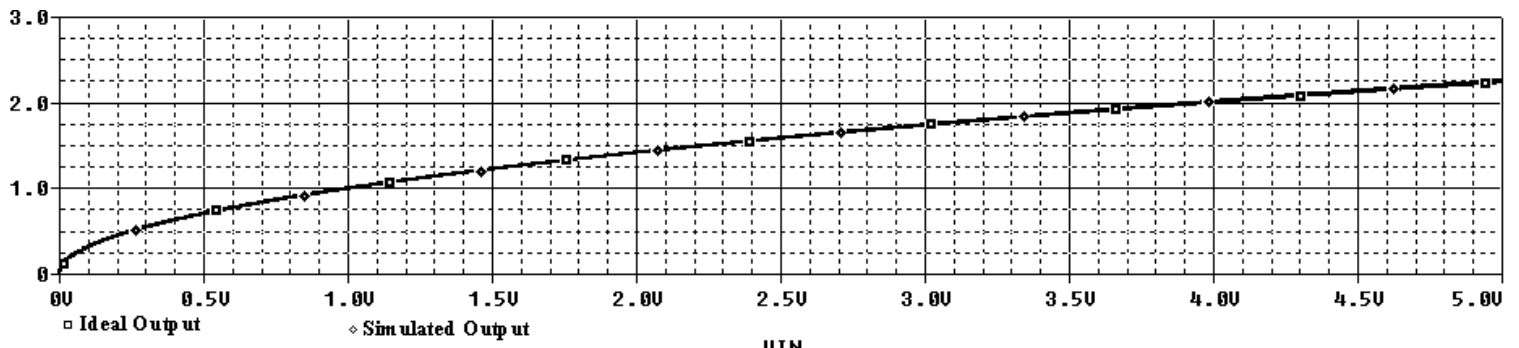

Figure 7. DC transfer characteristics of the Square rooting circuit 1

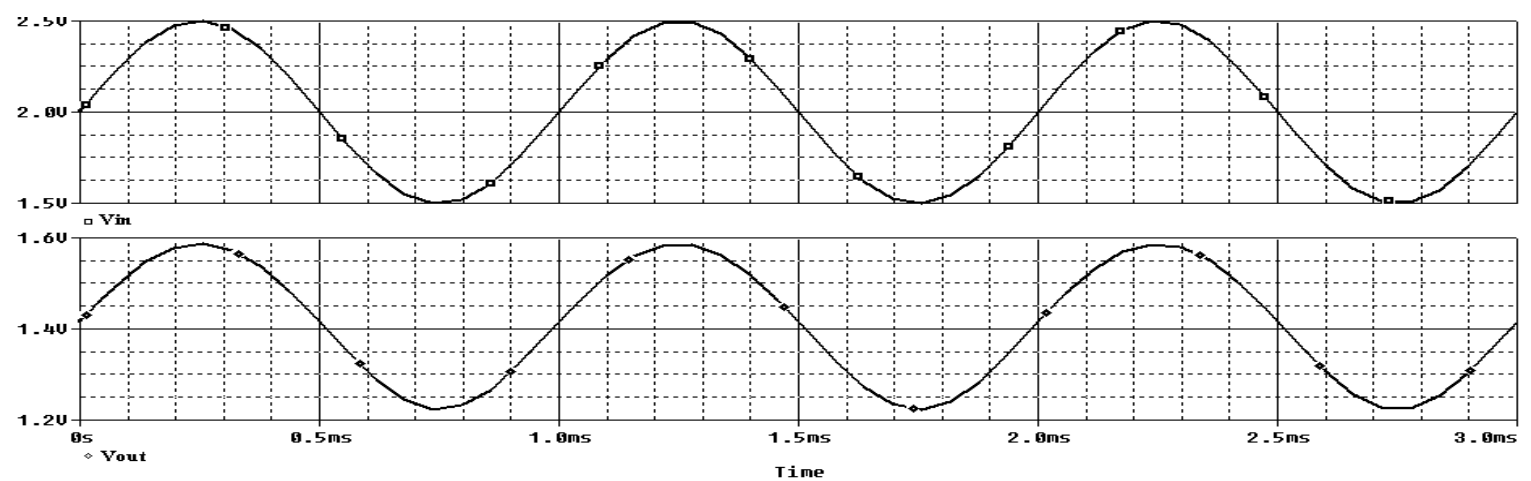

Figure 8. Output voltage waveform obtained from the Square rooting circuit 1 for $1 \mathrm{KHz}$ Sinusoidal input

In the simulations of the square rooting circuit 2 of Figure 4, CDBA from [3] was used operating at $\pm 12 \mathrm{~V}$ DC supply with the value of $R_{e q}$ made equivalent to $\sqrt{\frac{2 \alpha^{2} \beta_{p} L_{1}}{\left(K_{p} W_{2}+K_{n} W_{1} \beta_{p} \alpha^{2}\right)}}$ so that proportionality constant $\boldsymbol{e}$ becomes equal to 1 . By selecting $L_{1}=L_{2}=24 \mu \mathrm{m}$,
$W_{2}=40 \mu \mathrm{m}$ then $W_{1}=0.2455 W_{2}$ comes out to be equal to $81.45 \mu \mathrm{m}$. To obtain unity proportionality constant, the value of $R_{e q}$ was adjusted to $8722 \Omega$ by selecting $L_{3}=L_{4}=67 \mu \mathrm{m}, W_{3}=15 \mu \mathrm{m}, W_{4}=57.11 \mu \mathrm{m}, \mathrm{V}_{\mathrm{p}}=4 \mathrm{~V}$ and $\mathrm{V}_{\mathrm{n}}=-4 \mathrm{~V}$. The circuit of Figure 4 was also simulated using the same aspect ratios of MOS transistors and adjusted value of $R_{e q}$. Figure 9 shows the DC transfer characteristics of the square rooting circuit. Figure 10 
shows the transient response of the circuit to a sinusoidal input of $1 \mathrm{~V}_{\mathrm{pp}}$, average of $0.5 \mathrm{~V}$ and frequency of $1 \mathrm{kHz}$. Apart from the DC transfer characteristics and transient response of the proposed circuits, Table 1 shows the input voltage range, \% error for this range and the band width of the proposed circuits.

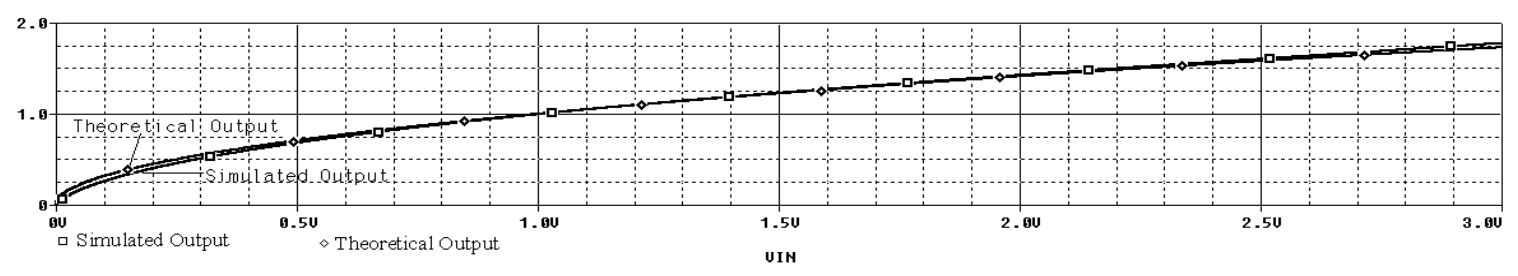

Figure 9. DC transfer characteristics of the square rooting circuit 2

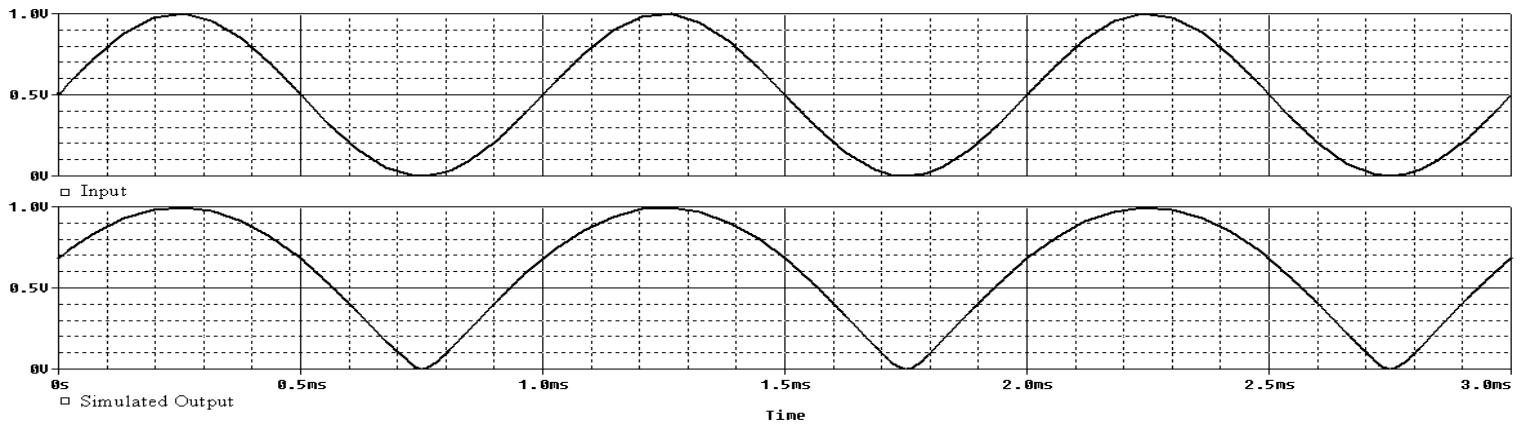

Figure 10. Output voltage waveform obtained from the square rooting circuit 2 for $1 \mathrm{KHz}$ sinusoidal input

Table 1. Input voltage range, \% error and band width of the proposed circuits

\begin{tabular}{|c|c|c|c|}
\hline Parameters & Squarer circuit & Square rooting circuit 1 & Square rooting circuit 2 \\
\hline Input voltage range (v) & -3.53 to 3.53 & 0 to 5.0 & 0.6 to 3.0 \\
\hline BError $=\left|\frac{I_{\text {output }}-S_{\text {output }}}{I_{\text {output }}}\right| \times 100 \%$ & Less than $2.5 \%$ & Less than $1.0 \%$ & $2.0 \%$ \\
\hline Band width (MHz) & 30.0 & 3.46 & 2.04 \\
\hline
\end{tabular}

\section{Comparison with the Existing CDBA- Based Squarer and Square Rooting Circuits}

Squarer circuit can also be realized from the four quadrant multiplier proposed by Keskin in [7] by taking two input signals to be same but such a realization will need an additional summer and difference circuits also. By contrast, in the proposed squarer circuit, only an additional inversion circuit is required. On the other hand, the current-mode squarer proposed by Lawanwisut and Siripruchyanun [9] needs four current-controlled CDBAs against one as required by the proposed squarer circuit. Furthermore, no square-rooting circuit has been reported earlier using CDBAs.

As compared to the squarer circuit based on Current Conveyors (CC) [12], the proposed squarer circuit has low output impedance to facilitate easy cascading without the need of additional buffers. Also, the band width of the proposed square-rooting circuits is $3.46 \mathrm{MHz}$ as compared to $400 \mathrm{kHz}$ for the CC-based square-rooting circuit of [12]. Furthermore, the proposed square-rooting circuit 1 employs only two active elements and the square-rooting circuit 2 employs only one active element as compared to three active elements (one CCII+ and two op-amps) used in the CC-based circuits of [12].

\section{Concluding Remarks}

New squarer and square-rooting circuits based on the CDBA have been proposed. These circuits exhibit wide input voltage range, smaller errors and have a low output impedance to facilitate easy cascading without requiring additional buffers. The proportionality constant of these circuits can be controlled by the value of an external resistor. Squarer circuit also works in trans-admittance mode. SPICE simulations have verified the workability of the proposed circuits.

\section{Acknowledgement}

This work was performed at the Analog Signal Processing Research Lab of NSIT, New Delhi.

\section{References}

[1] Ismail, M. "Analog VLSI: Signal and Information Processing (International Edition),” McGraw-Hill Inc. 1994.

[2] Roberts, G. W. and Sedra. A. S., "All current-mode frequency selective circuits,” Electronics Letters, 2. 759-761. 1989. 
[3] Acar, C. and Ozoguz, S., "A new versatile building block: current differencing buffered amplifier suitable for analog signal processing filters,” Microelectronics Journal, 30. 157-160. 1999.

[4] Acar, C. and Sedef, H., "Realization of $n^{\text {th }}$-order current transfer function using current-differencing buffered amplifiers," International Journal of Electronics, 90. 277-283. 2003

[5] Maheshwari, S. and Khan, I. A., "Current controlled current differencing buffered amplifier: Implementations and Applications," Active and Passive Electronic Components, 4. 219227. 2004.

[6] Pathak, J. K., Singh, A. K. and Senani, R., "Systematic realization of quadrature oscillators using current differencing buffered amplifiers,” IET Circuits, Devices and Systems, 5. 203-211. 2011.

[7] Keskin, A.U., "A Four quadrant analog multiplier employing single CDBA," Analog Integrated Circuits and Signal Processing, 40. 99-101. 2004.
[8] Siripruchyanun, M., "A Design of Analog Multiplier and Divider Using Current Controlled Current Differencing Buffered Amplifiers,” International Symposium on Integrated Circuits, 568571. 2007.

[9] Lawanwisut, S. and Siripruchyanun, M., "Temperature Insensitive Electronically Controllable Current-mode Squarer Based on CCCDBAs," Proceedings of the 1st International Conference on Technical Education (ICTE2009), 225-228. 2009.

[10] Al-Shahrani, S.M., "CMOS wideband auto-tuning phase shifter circuit,” IET, Electronics Letters, 43. 804-806. 2007.

[11] Senani, R. and Singh, A. K., "A new Universal Current mode biquad filter”, Frequenz, 56. 55-59. 2002.

[12] Liu, S.I., "Square-rooting and vector summation circuits using current conveyors," IEE Proc. Circuits, Devices and Systems. 142. 223-226. 1995. 